\title{
2 '-O methylation of the viral mRNA cap evades host restriction by IFIT family members
}

Stephane Daffis ${ }^{1 *}$, Kristy J. Szretter ${ }^{1 *}$, Jill Schriewer ${ }^{5}$, Jianqing Li $^{2}$, Soonjeon Youn ${ }^{1}$, John Errett ${ }^{6}$, Tsai-Yu Lin ${ }^{7}$, Stewart Schneller ${ }^{8}$, Roland Zust ${ }^{9}$, Hongping Dong ${ }^{11}$, Volker Thiel ${ }^{9,10}$, Ganes C. Sen ${ }^{12}$, Volker Fensterl ${ }^{12}$, William B. Klimstra ${ }^{13}$, Theodore C. Pierson ${ }^{7}$, R. Mark Buller ${ }^{4,5}$, Michael Gale Jr ${ }^{4,6}$, Pei-Yong Shi ${ }^{4,11}$ \& Michael S. Diamond ${ }^{1,2,3,4}$

Cellular messenger RNA (mRNA) of higher eukaryotes and many viral RNAs are methylated at the $N-7$ and $2^{\prime}-O$ positions of the $5^{\prime}$ guanosine cap by specific nuclear and cytoplasmic methyltransferases (MTases), respectively. Whereas N-7 methylation is essential for RNA translation and stability ${ }^{1}$, the function of $2^{\prime}-O$ methylation has remained uncertain since its discovery 35 years ago $^{2-4}$. Here we show that a West Nile virus (WNV) mutant (E218A) that lacks 2'-O MTase activity was attenuated in wild-type primary cells and mice but was pathogenic in the absence of type I interferon (IFN) signalling. 2' $\mathrm{O}$ methylation of viral RNA did not affect IFN induction in WNV-infected fibroblasts but instead modulated the antiviral effects of IFN-induced proteins with tetratricopeptide repeats (IFIT), which are interferon-stimulated genes (ISGs) implicated in regulation of protein translation. Poxvirus and coronavirus mutants that lacked $2^{\prime}$ - $O$ MTase activity similarly showed enhanced sensitivity to the antiviral actions of IFN and, specifically, IFIT proteins. Our results demonstrate that the $2^{\prime}-O$ methylation of the $5^{\prime}$ cap of viral RNA functions to subvert innate host antiviral responses through escape of IFIT-mediated suppression, and suggest an evolutionary explanation for $2^{\prime}-O$ methylation of cellular mRNA: to distinguish self from non-self RNA. Differential methylation of cytoplasmic RNA probably serves as an example for pattern recognition and restriction of propagation of foreign viral RNA in host cells.

Most eukaryotic mRNA contains a 5' Cap 0 (7mGpppN) structure with a methyl group at the N-7 position. In higher eukaryotes, methylation of cellular mRNA occurs additionally at the 2 '-O site of the penultimate $(7 \mathrm{mGpppNm}$, Cap 1$)$ and antepenultimate $(7 \mathrm{mGpppNmNm}$, Cap 2) $5^{\prime}$ nucleotides in the nucleus and cytoplasm, respectively ${ }^{3,5}$. Many viral mRNAs also contain Cap 1 and 2 structures, but cap acquisition occurs distinctly among virus families ${ }^{2,6}$. RNA and DNA viruses that replicate in the cytoplasm cannot use the host nuclear capping machinery, and thus have evolved MTases to facilitate N-7 and 2'-O capping or mechanisms to 'snatch' the cap from host cell mRNA ${ }^{1}$. It remains unclear how $2^{\prime}$ - $O$ methylation contributes to viral infection or cellular mRNA homeostasis ${ }^{2,3}$.

Flavivirus is a genus of positive-strand RNA viruses with a 5' Cap 1 structure that is generated by an MTase in the NS5 protein ${ }^{7}$. Whereas mutations abrogating the N-7 MTase activity abort WNV infection, an E218A substitution that completely abolished the $2^{\prime}-O$ but not $\mathrm{N}-7$ MTase activity (Supplementary Fig. 1) did not affect replication in permissive BHK cells ${ }^{8}$. Although $\mathrm{C} 57 \mathrm{BL} / 6$ mice infected subcutaneously with the parental WNV wild-type (WNV-WT) strain had an approximately $40 \%$ mortality rate, recipients of WNV-E218A showed
$0 \%$ mortality, even at high challenge doses (Fig. 1a, $P<0.05, n=10$ ) or after direct intracranial infection (Fig. 1c). Levels of WNV-E218A after subcutaneous inoculation were markedly decreased in the spleen, serum or brain compared with infection by WNV-WT (Fig. 1b).

Because dissemination of WNV-E218A was aborted in vivo, we assessed whether 2'-O methylation restricted the protective IFNinduced immune response. Mice lacking type I IFN signalling $\left(\right.$ Ifnar $1^{-/-}$) that were infected with WNV-WT showed $100 \%$ mortality and a mean time to death of 3.5 days, as seen previously ${ }^{9}$ (Fig. 1a). Remarkably, Ifnar ${ }^{-1-}$ mice infected with the WNV-E218A exhibited a similar phenotype with only a slightly delayed mean time to death of 4.5 days. Ifnar $1^{-1-}$ mice infected with WNV-E218A at day 3 sustained tissue titres that approached those of WNV-WT (Fig. 1d). Thus 2'-O methylation of WNV RNA is required for virulence in vivo, and its absence renders the virus sensitive to the IFN response.

Analysis of viral growth in primary mouse embryonic fibroblasts (MEFs) and macrophages $(\mathrm{M} \phi)$, which both produce and respond to type I IFN after WNV infection ${ }^{10}$, confirmed attenuation of WNVE218A in wild-type cells (50- and 151-fold lower at $72 \mathrm{~h}, P<0.05$, $n=3$ in MEF and $\mathrm{M} \phi$, respectively) and restored growth in Ifnar $1^{-1-}$ cells (Fig. 1e, f). Replication of WNV-E218A was also rescued in Irf3 ${ }^{-1-}$, $\operatorname{Irf} 3^{-1-} \times \operatorname{Irf} 7^{-1-}$ or IPS-1 $1^{-1-}$ cells that had altered or abolished IFN- $\alpha / \beta$ responses $^{11}$ (Supplementary Figs $2 \mathrm{a}-\mathrm{c}$ and $3 \mathrm{a}-\mathrm{d}$, respectively), but not in $\operatorname{Irf} 7^{-1-}$ or $T l r 3^{-1-}$ cells, which have normal IFN- $\beta$ or IFN- $\alpha$ and $-\beta$ responses after WNV infection, respectively ${ }^{10,12}$ (Supplementary Fig. 2d, e). These experiments confirmed that rescue of WNVE218A in primary cells requires attenuation of the IFN response.

Because 2'-O methylation rendered WNV-WT less susceptible to the IFN response than WNV-E218A, we hypothesized that it might directly limit IFN induction by affecting the avidity of viral RNA for the host sensor, RIG-I. However, direct binding assays with recombinant RIG-I and 2'-O unmethylated or methylated WNV RNA ( $5^{\prime}$ untranslated region) showed no change in binding (Supplementary Fig. 4). It remained possible that $2^{\prime}-O$ methylation of WNV RNA affected other proteins required for transcriptional activation of the IFN- $\beta$ gene. To evaluate this idea, Ifnar $1^{-1-}$ MEFs, which produce IFN- $\beta$ without responding to it, were infected at a high multiplicity of infection (MOI) and IFN- $\beta$ mRNA was measured. Notably, both WNV-WT and WNV-E218A stimulated IFN- $\beta$ transcription equivalently after infection (Fig. 2a). Thus a lack of $2^{\prime}$ - $O$ methylation does not affect pathogen sensing or IFN induction. To address whether $2^{\prime}-O$ methylation of viral RNA serves to antagonize or evade IFN effector functions, IPS-1 $1^{-1-}$ MEFs, which do not produce type I IFN after WNV infection but can respond to it ${ }^{11}$, were exposed to IFN- $\beta$ to

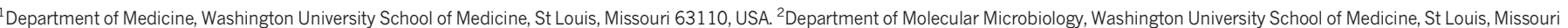

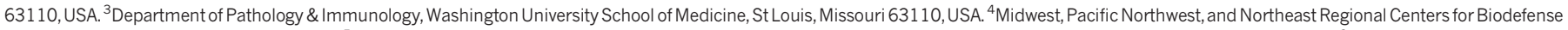

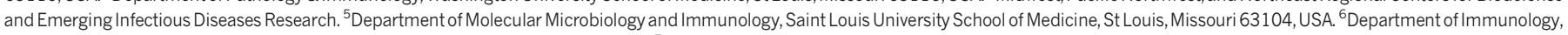

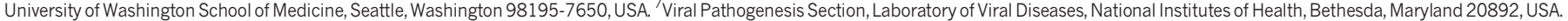

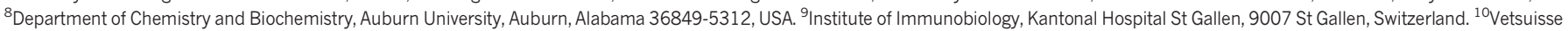

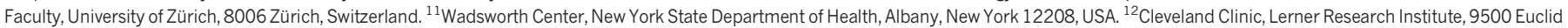
Avenue, Cleveland, Ohio 44195, USA. ${ }^{13}$ Department of Microbiology and Molecular Genetics, University of Pittsburgh Medical School, 3501 Fifth Avenue, Pittsburgh, Pennsylvania 15261, USA.

*These authors contributed equally to this work. 
a

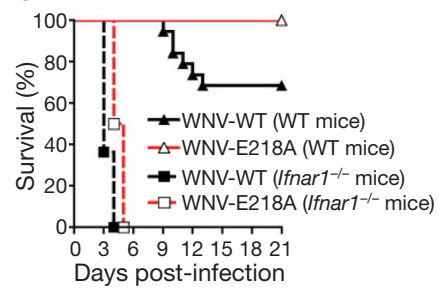

c

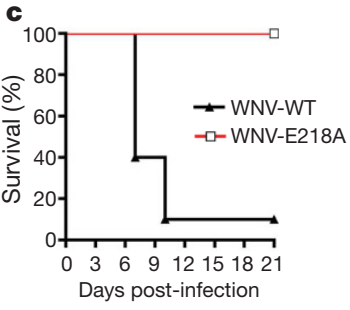

Fibroblasts

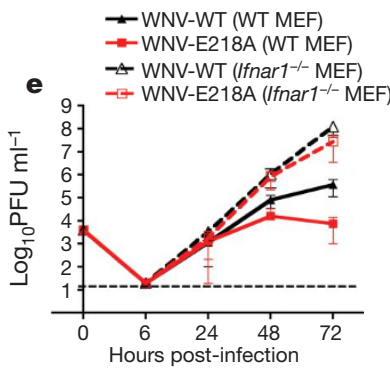

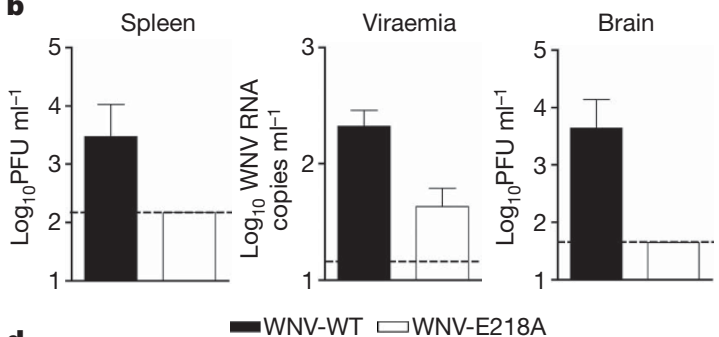

d

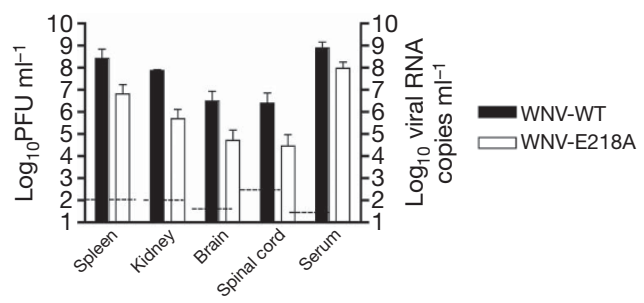

Macrophages

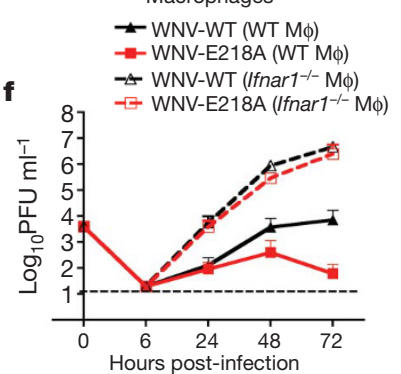

Figure $1 \mid$ WNV-E218A is attenuated in wild-type mice and cells but is virulent in Ifnar ${ }^{-1-}$ mice and cells. a, Survival curves of wild-type and Ifnar1 $^{-1-}$ C57BL/6 mice after subcutaneous infection with WNV-WT or WNV-E218A. b, Virus replication in wild-type mice in blood (day 4), spleen (day 4) or brain (day 8) after subcutaneous infection with WNV-WT or WNVE218A. c, Survival curves of wild-type mice after intracranial infection with
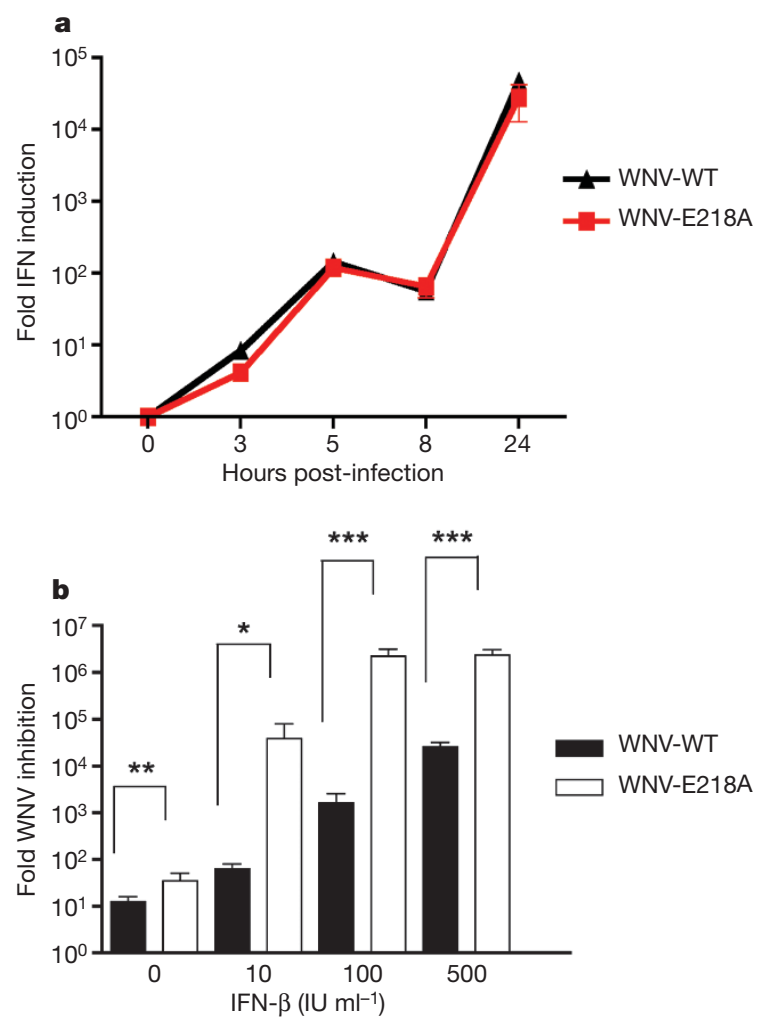

WNV-WT $\left(10^{1}\right)$ or WNV-E218A $\left(10^{5}\right.$ plaque-forming units (PFU)). d, Viral burden in the serum, spleen, kidney, spinal cord and brain from Ifnar $1^{-1-}$ mice at day 3 after infection. e, f, Replication of WNV-WT and WNV-E218A in wild-type or Ifnar1 ${ }^{-1-}$ MEFs (e) or M $\phi(\mathbf{f})$. Results are the average of three experiments performed in triplicate. Error bars, s.d.; dashed line, limit of sensitivity of the assay.

induce ISGs, and then infected. WNV-E218A displayed increased sensitivity to IFN- $\beta$ pretreatment compared with WNV-WT $(2,400,000$ - and 20,000-fold inhibition with 500 international units $\mathrm{ml}^{-1}$ of IFN- $\beta$, respectively) (Fig. $2 \mathrm{~b}$ ).

IFN induces hundreds of ISGs, some of which may have antiviral effector functions ${ }^{13}$. Among these, Ifit family members (for example, Ifit1 and Ifit2 (also known as ISG56 and ISG54, respectively)) are induced after WNV infection ${ }^{14}$, reduced in Irf3 $3^{-1-}$ and Ifnar $1^{-1-}$ cells (ref. 15 and Supplementary Fig. 5) and inhibit replication of some viruses ${ }^{16-18}$ in part, by interacting with eIF3 and limiting translation of viral $\mathrm{mRNA}^{19,20}$. To assess whether differential $2^{\prime}-O$ methylation of viral RNA might affect suppression by IFIT-1 and/or IFIT-2, we evaluated infection in 3T3 MEFs expressing a murine Ifit1 or Ifit2 transgene. As observed in primary cells, WNV-E218A replication in control 3T3 cells was reduced ( $~ 5$ - to 60 -fold decrease at $24-72 \mathrm{~h}, P<0.05, n=3)$ compared with WNV-WT, confirming that $2^{\prime}-O$ methylation is required for optimal infectivity (Fig. 3a). Transgenic expression of IFIT-2 reduced infection of WNV-WT ( 56- to 100-fold decrease at $24-72 \mathrm{~h}$, $P<0.0005, n=3$ ) (Fig. 3b) compared with replication in 3T3-green fluorescent protein (GFP) cells. In comparison, expression of IFIT-2

Figure 2 2 ' $-O$ methylation of viral RNA alters the sensitivity of WNV to the antiviral effects of IFN. a, IFN- $\beta$ gene induction in Ifnar ${ }^{-1-}$ MEF after WNV-WT or WNV-E218A infection. Results are representative of three independent experiments performed in duplicate. b, Viral replication in IPS$1^{-1-}$ MEF after IFN- $\beta$ pretreatment. The data are the average of two independent experiments performed in triplicate, and the asterisks indicate differences that are statistically significant $(* * * P<0.0001$; $* * P<0.005$; $* P<0.05)$. Error bars, s.d. IU, international units. 
a

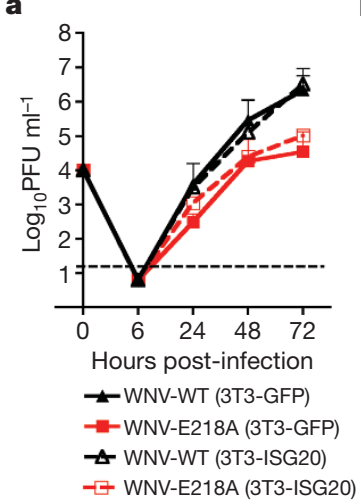

b

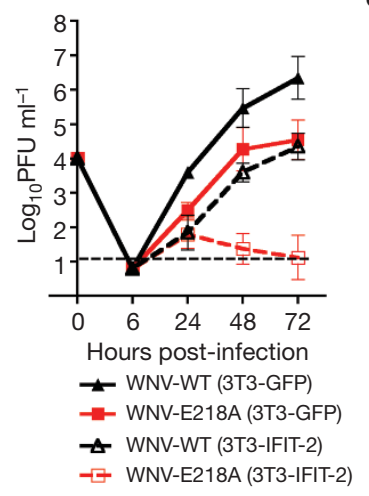

d

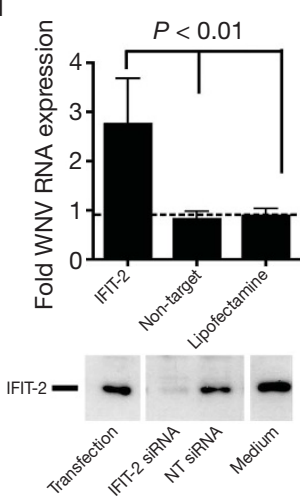

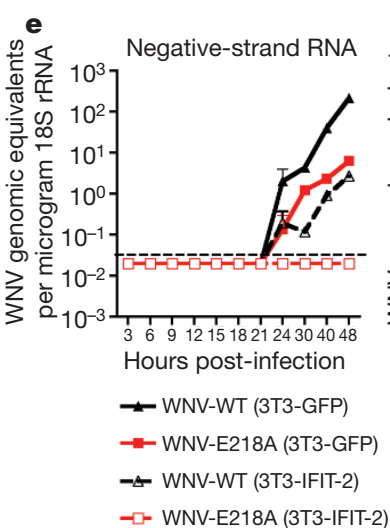

Figure $3 \mid$ WNV-E218A is more sensitive to the antiviral actions of Ifit genes. a-c, Viral replication of WNV-WT or WNV-E218A in 3T3 MEFs transgenically expressing GFP (a-c), ISG20 (a), IFIT-2 (b) or IFIT-1 (c). The data are the average of three experiments performed in duplicate. d, siRNA knockdown of IFIT-2 enhances replication of WNV-E218A. 3T3 cells were transfected with a non-target (NT) or IFIT-2 siRNA and then infected with WNV-E218A. One day post-infection cells were collected and (top) viral RNA was assayed by quantitative reverse transcriptase PCR. The data are the average

virtually abolished replication of WNV-E218A (up to 2,700-fold decrease at $72 \mathrm{~h}, P<0.0005, n=3$ ) (Fig. 3b). Expression of IFIT-1 in $3 \mathrm{~T} 3$ cells had minimal inhibitory effects on WNV infection (Fig. 3c). To confirm the linkage between IFIT-2 expression and restriction of infection, short interfering RNA (siRNA) knockdown experiments were performed. Transfection of a sequence-specific siRNA that reduced protein expression of IFIT-2 enhanced replication of WNV-E218A $(P<0.01, n=3)$ (Fig. 3d). These experiments demonstrate that mouse IFIT-2 is an antiviral effector of IFN actions, whose inhibitory activity is minimized by 2'-O methylation of viral RNA.

Although IFIT family orthologues exist over a broad evolutionary time-frame ${ }^{21}$, humans have a distinct complement of Ifit genes (IfitI (ISG56), Ifit2 (ISG54), Ifit3 (ISG60) and Ifit5 (ISG58)). Transient transgenic expression of human IFIT- 5 but not IFIT-1, IFIT- 2 or IFIT-3 in human $293 \mathrm{~T}$ cells inhibited infection of WNV-E218A $(P=0.003$, $n=3$ ) (Supplementary Fig. 6), which suggests a species-specificity of Ifit genes in restricting WNV lacking 2'-O methylated RNA.

We assessed the stage of the WNV life cycle that was restricted by mouse IFIT-2. Using strand-specific quantitative reverse transcriptase PCR to quantify genomic (positive strand) and replicative intermediate (negative strand) viral RNA, we found that in control 3T3 cells each increased by $18 \mathrm{~h}$ after infection (Fig. 3e, f), whereas the expression of mouse IFIT- 2 delayed production of both by approximately $15 \mathrm{~h}$ in the context of WNV-WT infection. In comparison, increases in negative and positive strand RNA were abolished in IFIT-2 transgenic cells infected with WNV-E218A. The levels of WNV-E218A positivestrand RNA remained essentially constant over the time course, c

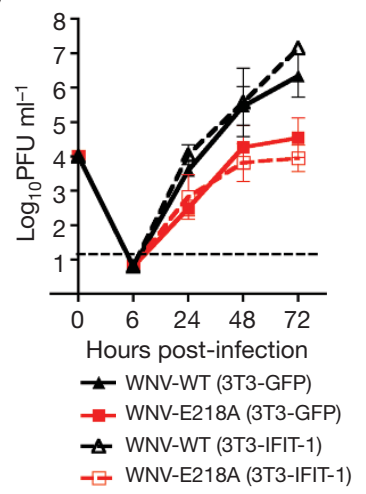

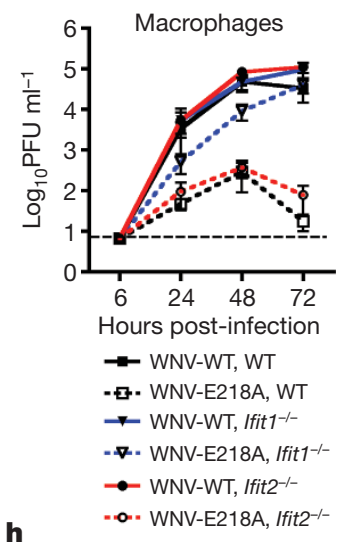

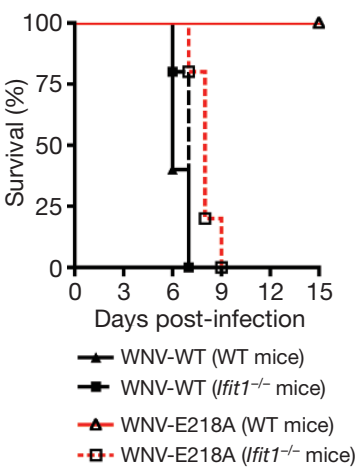

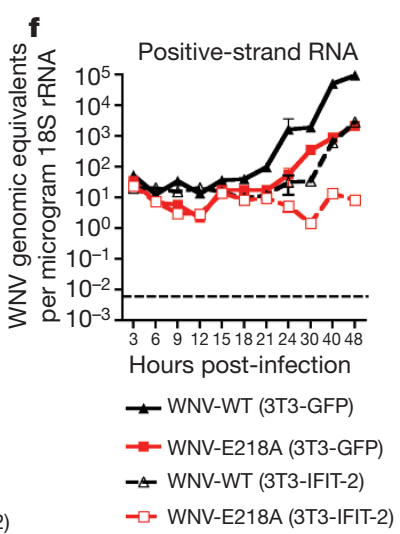

of three experiments performed in duplicate. Bottom, knockdown of IFIT-2 protein was confirmed by western blot. e, f, Murine IFIT-2 expression prevents accumulation of negative- and positive-strand viral RNA in WNV-E218Ainfected cells. g, Replication of WNV-E218A is attenuated in wild-type and Ifit $2^{-/-} \mathrm{M} \phi$ but restored in Ifit $1^{-/-}$cells. $\mathbf{h}$, Survival curves of wild-type or Ifit ${ }^{-/-}$mice after intracranial challenge with $10^{5}$ plaque-forming units of WNV-WT or WNV-E218A. Error bars, s.d.; dashed line, limit of sensitivity of the assay.

suggesting that the lack of $2^{\prime}-O$ methylation did not affect viral RNA stability. Thus mouse IFIT-2 blocks infection of the E218A mutant in fibroblasts at or before negative-strand synthesis.

As other virus families encode $2^{\prime}$-O MTases, we sought to determine if 2'-O-methylation-dependent evasion of IFIT proteins functions as a more general immune escape mechanism. We obtained a vaccinia virus (VACV) mutant (J3-K175R) that lacked 2'-O MTase activity, replicated normally in BSC40 cells $\mathrm{s}^{22}$ but was attenuated in wild-type $\mathrm{M} \phi$ (approximately six- to eightfold reduction at $24-72 \mathrm{~h}$ ) and fully rescued in Ifnar1 $1^{-1-} \mathrm{M} \phi$ (Fig. 4a). Growth curves with VACV-WT and VACVJ3-K175R in 3T3 cells expressing GFP or ISG20 confirmed an essential role of $2^{\prime}$ - $O$ methylation in poxvirus infection (approximately three- to fivefold reduction at 24-72 h, $P<0.005, n=3$ ) (Fig. 4b). Transgenic expression of IFIT-2, however, did not affect replication of VACV-WT $(P>0.5, n=3)$, which suggests that IFIT-2 lacks activity against VACV-WT or that the virus efficiently antagonizes its antiviral effect. Expression of mouse IFIT-2 but not IFIT- 1 further reduced infection of VACV-J3-K175R (6- to 25-fold decrease, $P<0.01, n=3$ ) (Fig. 4c, d). Consistent with these findings, wild-type C57BL/6 mice were resistant to lethal challenge with VACV-J3-K175R ( $0 \%$ lethality, $n=6)$ but sensitive to infection with VACV-WT (100\% lethality, $n=13)$. In contrast, in Ifnar ${ }^{-1-}$ mice, VACV-J3-K175R was virulent as all animals succumbed to infection with similar kinetics compared with those infected with VACV-WT (Supplementary Fig. 7).

We examined the replication of a wild type and 2'-O MTase mutant (D130A in the nsp16 protein ${ }^{23}$ of mouse hepatitis virus (MHV). MHV-D130A was more sensitive to the effects of IFN- $\beta$ pretreatment 
a

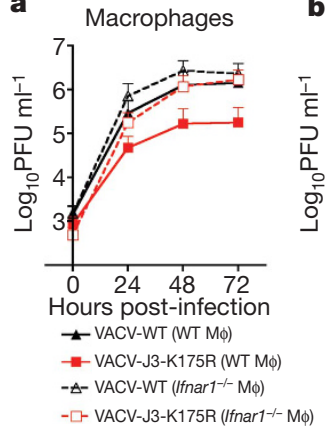

b

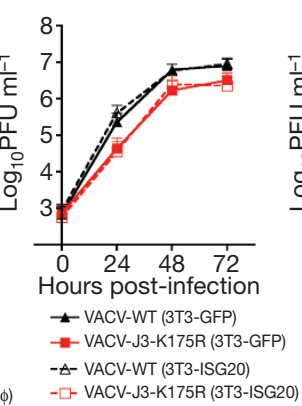

C

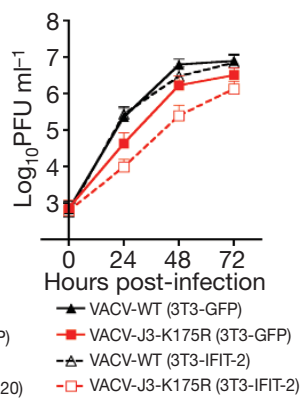

d

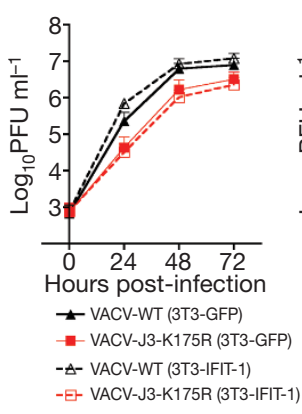

e

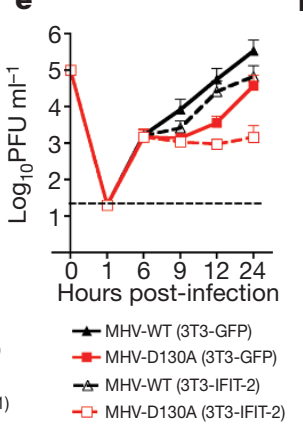

'

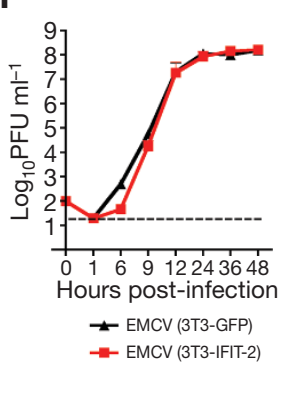

Figure $4 \mid$ Poxvirus and coronavirus mutants lacking $2^{\prime}$ - $O$ methylation are more sensitive to the antiviral effects of murine IFIT-2. a-d, Studies with VACV. a, Viral replication of VACV-WT or VACV-J3-K175R in wild-type or Ifnarl $^{-1-} \mathrm{M} \phi$ (a) or 3T3 MEF expressing GFP (b-d), ISG20 (b), Ifit2 (c) or Ifit1 (d). e, Viral replication of MHV-WT or MHV-D130A in 3T3 cells expressing GFP or IFIT-2. f, Viral replication of EMCV in 3T3 cells expressing GFP or IFIT-2. Error bars, s.d.; dashed line, limit of sensitivity of the assay.

(Supplementary Fig. 8), attenuated in control 3T3 cells (approximately 6 - to 15 -fold reduction at 9-24 h, $P<0.05, n=3$ ) (Fig. $4 \mathrm{e}$ ), and sensitive to transgenic expression of mouse IFIT-2 (approximately 8 - to 234-fold reduction, $P<0.05, n=3$ ) compared with MHV-WT (approximately two- to fivefold decrease at 9-24 h, $P<0.05, n=3$ ). Thus, analogous to flaviviruses and poxviruses, the 2 ' $-O$ methylation of coronavirus RNA supports evasion from the antiviral effects of IFIT-2. In contrast, transgenic expression of IFIT-2 did not affect replication of a picornavirus, which lacks a $5^{\prime}$ cap structure (Fig. 4f).

To confirm the role of IFIT proteins in restricting viruses lacking $2^{\prime}$ $O$ methylation, growth curves were performed in wild-type, Ifit $1^{->}$or Ifit $2^{-/-} \mathrm{M} \phi$. Surprisingly, the infectivity of WNV-E218A was almost completely rescued in IFIT- $1^{-1-} \mathrm{M} \phi(2,300$-fold increase in titre at $72 \mathrm{~h}, P<0.04$ ) but not in Ifit $2^{-/-} \mathrm{M} \phi$ (Fig. 3g), and the virulence of WNV-E218A was almost entirely restored in Ifit $1^{-/-}$mice (Fig. $3 \mathrm{~h}$ ). Thus, in primary $\mathrm{M} \phi$ and in mice, IFIT-1 plays a dominant role in restricting infection of WNV lacking 2'-O methylation.

We demonstrate that among unrelated RNA and DNA viruses that replicate in the cytoplasm and contain $5^{\prime}$ cap structures, $2^{\prime}$ - $O$ methylation of viral RNA enhances virulence through evasion of intrinsic cellular defence mechanisms. 2'-O methylation of cellular RNA may have evolved as a means of distinguishing self from non-self RNA by the host during virus infection. Induction of Ifit family genes, several of which attenuate translation ${ }^{19,20,24}$, could preferentially recognize viral mRNA lacking 2'-O methylation and selectively restrict propagation. Plants, which lack an IFN response network or Ifit family member orthologues, and their viruses, accordingly lack $2^{\prime}$-O-methylation of mRNA. Given that host $2^{\prime}-O$ methylation of cellular mRNA largely occurs in the nucleus, pharmacological strategies that disrupt cytoplasmic 2'-O MTase activity could represent a novel class of therapy against several globally relevant pathogenic viruses that replicate exclusively in the cytoplasm.

\section{METHODS SUMMARY}

Viruses. WNV-WT and WNV-E218A were propagated in BHK21 cells as described $^{8}$. VACV-WT and VACV-J3-K175R ${ }^{22}$ (a gift from R. Condit) and encephalomyocarditis virus (EMCV) (strain K) were propagated in HeLa and L929 cells, respectively. Generation of MHV-WT (strain A59) and MHV-D130A recombinant coronaviruses has been described ${ }^{25}$.

Mouse experiments. C57BL/6 wild-type and immunodeficient $\left(\right.$ Ifnar ${ }^{-1-}$, Ifit $1^{-1-}$, Ifit $2^{-1-}, \operatorname{Irf3} 3^{-1-}, \operatorname{Irf7}^{-1-}, \operatorname{Irf3} 3^{-1-} \times \mathrm{Irf7}^{-1-}$ and $\left.I P S-1^{-1-}\right)$ mice were bred at Washington University. Infection experiments were performed with approval of the Washington University and St Louis University Animal Studies Committees. Viral titres in blood and organs were quantified as previously described ${ }^{11}$.

Cell culture and viral infection. Bone-marrow-derived $M \phi$ and MEF were generated as described ${ }^{11}$. 3T3 fibroblasts expressing GFP or ISG were previously described ${ }^{18}$. Cells were infected with WNV, VACV, MHV or EMCV at MOIs of $0.01,1,1$ and 0.001, respectively. Lysates or supernatants were titred by plaque assay on BHK21-15 cells for WNV and EMCV, BSC-1 cells for VACV and L929 cells for MHV.

Quantification of IFN- $\boldsymbol{\beta}$ mRNA. Ifnar $1^{-/-}$MEFs were infected at an MOI of 10 with WNV-WT or WNV-E218A. Total RNA was isolated, treated with DNase (Qiagen), and IFN- $\beta$ mRNAs were amplified by quantitative reverse transcriptase PCR as described previously ${ }^{11}$.

IFN- $\beta$ pretreatment experiment. IPS- $1^{-/-}$MEFs were pretreated with increasing doses of mouse IFN- $\beta$ (PBL Laboratories) for $24 \mathrm{~h}$ and then infected with WNV or MHV at an MOI of 0.1. Supernatants were collected at 48 or $12 \mathrm{~h}$ after infection, respectively, and titred by plaque assay.

Strand-specific real-time reverse transcriptase PCR. Quantification of positiveand negative-strand WNV RNA was performed using a T7-tagged primer strategy ${ }^{9}$. Fibroblasts expressing GFP or mouse IFIT-2 were infected with WNV-WT or WNV-E218A at an MOI of 1 and total RNA was collected at indicated time points.

\section{Received 24 February; accepted 13 September 2010.}

1. Furuichi, Y. \& Shatkin, A. J. Viral and cellular mRNA capping: past and prospects. Adv. Virus Res. 55, 135-184 (2000).

2. Wei, C. M. \& Moss, B. Methylated nucleotides block 5 '-terminus of vaccinia virus messenger RNA. Proc. Natl Acad. Sci. USA 72, 318-322 (1975).

3. Wei, C. M., Gershowitz, A. \& Moss, B. Methylated nucleotides block 5 ' terminus of HeLa cell messenger RNA. Cell 4, 379-386 (1975).

4. Muthukrishnan, S., Moss, B., Cooper, J. A. \& Maxwell, E. S. Influence of 5'-terminal cap structure on the initiation of translation of vaccinia virus mRNA. J. Biol. Chem. 253, 1710-1715 (1978).

5. Langberg, S. R. \& Moss, B. Post-transcriptional modifications of mRNA. Purification and characterization of cap I and cap II RNA (nucleoside-2'-)methyltransferases from HeLa cells. J. Biol. Chem. 256, 10054-10060 (1981)

6. Fechter, P. \& Brownlee, G. G. Recognition of mRNA cap structures by viral and cellular proteins. J. Gen. Virol. 86, 1239-1249 (2005).

7. Dong, H., Zhang, B. \& Shi, P. Y. Flavivirus methyltransferase: a novel antiviral target Antiviral Res. 80, 1-10 (2008).

8. Zhou, Y. et al. Structure and function of flavivirus NS5 methyltransferase. J. Virol. 81, 3891-3903 (2007).

9. Samuel, M. A. \& Diamond, M. S. Alpha/beta IFN protects against lethal West Nile virus infection by restricting cellular tropism and enhancing neuronal survival. J. Virol. 79, 13350-13361 (2005).

10. Daffis, S. et al. Interferon regulatory factor IRF-7 induces the antiviral alpha interferon response and protects against lethal West Nile virus infection. J. Virol. 82, 8465-8475 (2008).

11. Daffis, S., Suthar, M. S., Szretter, K. J., Gale, M. Jr \& Diamond, M. S. Induction of IFN- $\beta$ and the innateantiviral response in myeloid cells occurs throughan IPS-1-dependent signal that does not require IRF-3 and IRF-7. PLoS Pathog. 5, e1000607 (2009).

12. Daffis, S., Samuel, M. A., Suthar, M. S., Gale, M. Jr \& Diamond, M. S. Toll-like receptor 3 has a protective role against West Nile virus infection. J. Virol. 82, 10349-10358 (2008).

13. Der, S. D., Zhou, A., Williams, B. R. \& Silverman, R. H. Identification of genes differentially regulated by interferon $\alpha, \beta$, or $\gamma$ using oligonucleotide arrays. Proc. Natl Acad. Sci. USA 95, 15623-15628 (1998).

14. Wacher, C. et al. Coordinated regulation and widespread cellular expression of interferon-stimulated genes (ISG) ISG-49, ISG-54, and ISG-56 in the central nervous system after infection with distinct viruses. J. Virol. 81, 860-871 (2007).

15. Daffis, S., Samuel, M. A., Keller, B. C., Gale, M. Jr \& Diamond, M. S. Cell-specific IRF-3 responses protect against West Nile virus infection by interferon-dependent and independent mechanisms. PLoS Pathog. 3, e106 (2007).

16. Fensterl, V., White, C. L., Yamashita, M. \& Sen, G. C. Novel characteristics of the function and induction of murine p56 family proteins. J. Virol. 82, 11045-11053 (2008).

17. Sumpter, R. Jr et al. Regulating intracellular antiviral defense and permissiveness to hepatitis $C$ virus RNA replication through a cellular RNA helicase, RIG-I. J. Virol. 79, 2689-2699 (2005).

18. Zhang, Y., Burke, C. W., Ryman, K. D. \& Klimstra, W. B. Identification and characterization of interferon-induced proteins that inhibit alphavirus replication. J. Virol. 81, 11246-11255 (2007). 


\section{RESEARCH LETTER}

19. Terenzi, F. Hui, D. J. Merrick, W. C. \& Sen, G. C. Distinct induction patterns and functions of two closely related interferon-inducible human genes, ISG54 and ISG56. J. Biol. Chem. 281, 34064-34071 (2006).

20. Hui, D. J., Terenzi, F., Merrick, W. C.\& Sen, G. C. Mouse p56 blocks a distinct function of eukaryotic initiation factor 3 in translation initiation. J. Biol. Chem. 280, 3433-3440 (2005)

21. Sarkar, S. N. \& Sen, G. C. Novel functions of proteins encoded by viral stressinducible genes. Pharmacol. Ther. 103, 245-259 (2004).

22. Latner, D. R., Thompson, J. M., Gershon, P. D., Storrs, C. \& Condit, R. C. The positive transcription elongation factor activity of the vaccinia virus $\mathrm{J} 3$ protein is independent from its (nucleoside-2'-O-) methyltransferase and poly(A) polymerase stimulatory functions. Virology 301, 64-80 (2002).

23. Decroly, E. et al. Coronavirus nonstructural protein 16 is a cap-0 binding enzym possessing (nucleoside-2'0)-methyltransferase activity. J. Virol. 82, 8071-8084 (2008).

24. Hui, D. J., Bhasker, C. R., Merrick, W. C. \& Sen, G. C. Viral stress-inducible protein p56 inhibits translation by blocking the interaction of elF3 with the ternary complex elF2.GTP.Met-tRNAi. J. Biol. Chem. 278, 39477-39482 (2003).

25. Coley, S. E. et al. Recombinant mouse hepatitis virus strain A59 from cloned, fulllength cDNA replicates to high titers in vitro and is fully pathogenic in vivo. J. Virol. 79, 3097-3106 (2005).
Supplementary Information is linked to the online version of the paper at www.nature.com/nature.

Acknowledgements This work was supported by National Institutes of Health grants U54 AI081680, U19 AI083019 and R01 Al074973 (to M.G. and M.S.D.), R01 Al56540 (to S.S.), U54 AI057160 (to R.M.B.) and U54 AI057158 (to P.Y.S.), R01 CA068782 (to G.C.S.) the Swiss National Science Foundation, 3100A0-118425/1, and the Novartis Foundation (to V.T. and R.Z.). We thank H. Virgin and B. Moss for reading the manuscript.

Author Contributions S.D., K.J.S., R.M.B., T.C.P., M.G., P.-Y.S. and M.S.D. designed the experiments. S.D., K.J.S., J.S., J.L., T.-Y.L. and H.D. performed the experiments. S.D., K.J.S., J.S., R.M.B., M.G., P.-Y.S. and M.S.D. analysed the data. S.Y., V.F., G.C.S., W.B.K., R.Z and V.T. provided key reagents and expertise. S.D., K.J.S., R.M.B, T.C.P., M.G., P.-Y.S. and M.S.D. wrote and edited the manuscript.

Author Information Reprints and permissions information is available at www.nature.com/reprints. The authors declare no competing financial interests. Readers are welcome to comment on the online version of this article at www.nature.com/nature. Correspondence and requests for materials should be addressed to M.S.D. (diamond@borcim.wustl.edu). 